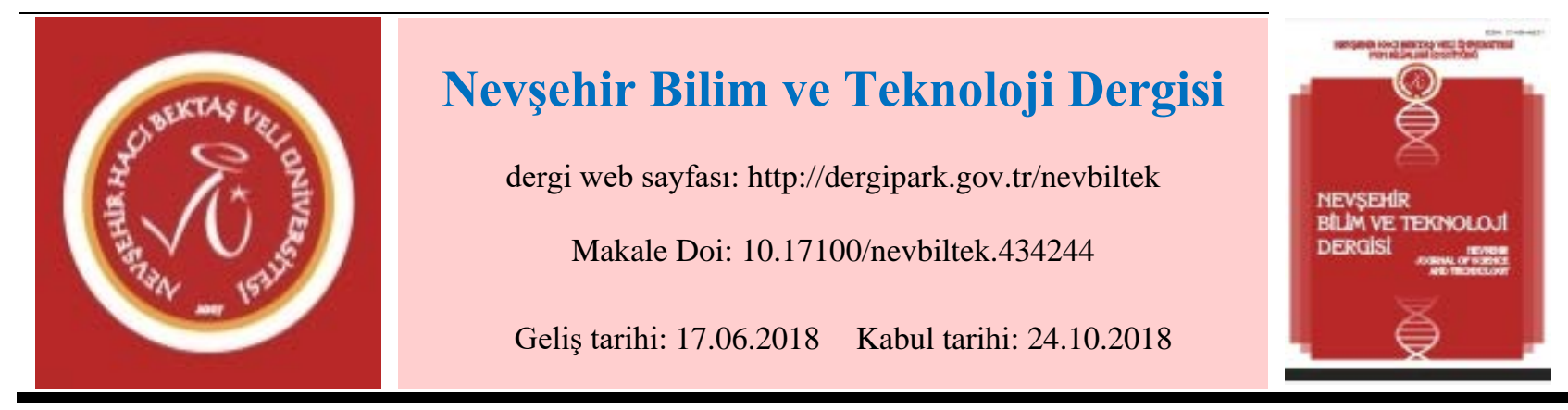

\title{
Eu-Dy Katkılı Stronsiyum Alüminat/Epoksi Kompozit Filmlerin Uygulanması ${ }^{1}$
}

\author{
Atilla Evcin ${ }^{1}$, Süleyman Akpınar ${ }^{1}$, Nalan Çiçek Bezir ${ }^{2}$ \\ ${ }^{1}$ Afyon Kocatepe Üniversitesi, Mühendislik Fakültesi, Malzeme Bilimi ve Mühendisliği Bölümü, Afyon \\ ORCID ID: 0000-0002-0163-5097 \\ ORCID ID: 0000-0002-7959-3464
}

${ }^{2}$ Süleyman Demirel Üniversitesi, Fen Edebiyat Fakültesi, Fizik Bölümü, Isparta

ORCID ID: 0000-0002-5708-1521

\begin{abstract}
$\ddot{\mathrm{Oz}}$
Luminesans malzemelerden birisi de Stronsiyum Alüminattır. Faz diyagramından 5 farkl ikili faz $\left(\mathrm{Sr}_{4} \mathrm{Al}_{2} \mathrm{O}_{7}, \mathrm{Sr}_{3} \mathrm{Al}_{2} \mathrm{O}_{6}, \mathrm{SrAl}_{2} \mathrm{O}_{4}, \mathrm{SrAl}_{4} \mathrm{O}_{7}\right.$ ve $\left.\mathrm{SrAl}_{2} \mathrm{O}_{19}\right)$ elde edilebilir. Bunlar arasında $\mathrm{SrAl}_{2} \mathrm{O}_{4}$ mükemmel kimyasal kararlılık ve uzun süreli 1şıldamaya sahiptir. Daha fazla ışıldamaya sahip olması için farklı katkılar kullanılmıştır. Bu katkılar içerisinde en etkili olanı nadir toprak elementlerinden Eu ve Dy’dir. Bu çalışmada Eu-Dy katkılı Stronsiyum alüminat tozları sol-jel yöntemiyle hazırlanmıştır. $\mathrm{SrAl}_{2} \mathrm{O}_{4}$ oluşturabilmek için uygun başlangıç kimyasalları (Stronsiyum nitrat, Alüminyum nitrat) seçilerek, çözeltileri hazırlanmıştır. İki çözeltinin karıştırılmasıyla oluşan çözeltiye $0.01 \mathrm{~mol} \%$ Disprozyum nitrat ve Evropyum nitrat eklenmiş̧ir. Oda sıcaklığında jelleşen tozlar $180^{\circ} \mathrm{C}$ 'de 24 saat etüvde bekletilmiştir. Kurutulan tozlar $1200{ }^{\circ} \mathrm{C}$ 'de 4 saat sinterlenmiştir. $\mathrm{SrAl}_{2} \mathrm{O}_{4}: \mathrm{Eu}^{2+}, \mathrm{Dy}^{3+}$ tozların termal, morfolojik, kristaloğrafik özellikleri sırasıyla termo gravimetrik/diferansiyel taramalı kalorimetri (DSC/TGA), taramalı elektron mikroskopu (SEM), X-ışını difraksiyonu (XRD) kullanılarak karakterize edilmiștir. Tozların $\mathrm{SrAl}_{2} \mathrm{O}_{4}$ yapısında olduğu ve herhangi bir istenmeyen fazın bulunmadığ 1 görülmüştür. DSC/TGA sonucu $800^{\circ} \mathrm{C}$ 'den sonra herhangi bir ekzotermik veya endotermik reaksiyon ile bir ağırlık kaybı kaydedilmemiştir. $\mathrm{Bu} \mathrm{SrAl}_{2} \mathrm{O}_{4}: \mathrm{Eu}^{2+}, \mathrm{Dy}^{3+}$ tozların epoksi reçine ile kompoziti hazırlanmış ve seramik yüzeylere uygulanmıştır. Uygulanan kompozitin uzun süreli 1ş1ldama gösterdiği görülmüştür.
\end{abstract}

Anahtar Kelimeler: Luminesans, Stronsiyum alüminat, nadir toprak elementleri, sol-jel

\section{Application of Eu-Dy Doped Strontium Aluminate/Epoxy Composite Films}

\begin{abstract}
One of the luminescent materials is the Strontium Aluminate. From the phase diagram, 5 different binary phases $\left(\mathrm{Sr}_{4} \mathrm{Al}_{2} \mathrm{O}_{7}, \mathrm{Sr}_{3} \mathrm{Al}_{2} \mathrm{O}_{6}, \mathrm{SrAl}_{2} \mathrm{O}_{4}\right.$, $\mathrm{SrAl}_{4} \mathrm{O}_{7}$ ve $\mathrm{SrAl}_{2} \mathrm{O}_{19}$ ) can be obtained. Among them, $\mathrm{SrAl}_{2} \mathrm{O}_{4}$ has excellent chemical stability and long-lasting luminescence. Different additives have been used to have more luminescence. Among these additives, Eu and Dy are the most effective and rare earth elements. In this study, Eu-Dy doped strontium aluminate powders were prepared by sol-gel method. To fabricate $\mathrm{SrAl}_{2} \mathrm{O}_{4}$, proper chemical precursors (Strontium nitrate, Aluminum nitrate) were chosen and their solutions were prepared. $0.01 \mathrm{~mol} \%$ of Dysprosium nitrate and Europium nitrate was added to the solution formed by mixing their solutions. Gelling powders at room temperature were dried in the oven at $180^{\circ} \mathrm{C}$ for 24 hours. Dried powders were sintered at $1200^{\circ} \mathrm{C}$ for 4 hours. Thermal, morphological and crystallographic properties of $\mathrm{SrAl}_{2} \mathrm{O}_{4}: \mathrm{Eu}^{2+}, \mathrm{Dy}^{3+}$ powders were characterized by using thermogravimetric/differential scanning calorimetry (DSC/TGA), X-ray diffraction (XRD) and scanning electron microscopy (SEM) techniques, respectively. It was observed that the powders were of $\mathrm{SrAl} 2 \mathrm{O} 4$ structure and no undesirable phase was present. $\mathrm{SrAl}_{2} \mathrm{O}_{4}$ : $\mathrm{Eu}^{2+}$, $\mathrm{Dy}^{3+}$ powders and epoxy resin composites were prepared and applied on ceramic surfaces. It was observed that the applied composite showed long-lasting luminescence.
\end{abstract}

Keywords: Luminescence, Strontium aluminate, Rare earth element, sol-gel

\footnotetext{
${ }^{1} \mathrm{Bu}$ çalışma The Internatinonal Conference on Materials Science Mechanical and Automotive Engineerings and Technology in ÇEŞME/IZZMIR (IMSMATEC'18) de bildiri olarak sunulmuştur.

Sorumlu yazar e-mail: evcin@aku.edu.tr
} 


\section{Giriş}

Kararlı düşük enerjili durumda bulunan bir maddenin atom veya molekülleri herhangi bir kaynaktan gelen enerjinin soğurulması sonucu uyarılarak, yüksek enerjili kararsız hale geçer ve atom veya moleküller, kazandıkları bu fazla enerjiyi atarak temel hale dönmek isterler. Uyarılmış atom veya moleküller elektromanyetik ışınım yaparak temel enerji seviyelerine dönerler ki, buna "Lüminesans” denir [1]. Fosfor malzemeleri mor ötesi 1şınlara tabi tutulduklarında kırmızı, mavi ve yeşil renklerde görülebilen ışıldama yaparlar. Elektronların daha yüksek bir enerji seviyesine uyarılması sonucu gözlemlenir. Bu gibi uyarmalar değişik yöntemlerle de yapılabilmektedir [2,3]. Uyarılma şekline göre lüminesans mekanizmaları Tablo 1'de gösterilmiştir [4].

Tablo 1. Uyarılma şekline göre oluşan lüminesans mekanizmaları

\begin{tabular}{ll} 
Işıma Türü & Uyarılma Türü \\
\hline Fotolüminesans (Floresans, fosforesans) & Fotonların absorblanması \\
& X ışınları, $\alpha, \beta, \gamma$ ışıması \\
Katodolüminesans & Katod Işılları (elektron demeti) \\
Elektrolüminesans & Elektriksel Alan \\
Termolüminesans & Radyooaktif Işılar \\
Kemilüminesans & Kimyasal Prosesler(oksidasyon gibi) \\
Biolüminesans & Biokimyasal Prosesler
\end{tabular}

Uyarılmayı sağlayan enerji eğer kimyasal bir reaksiyondan karşılanmışsa buna kemilüminesans, elektrot reaksiyonundan sağlanmışsa elektrolüminesans, ısıtarak sağlanırsa termolüminesans ve eğer fotonların absorblanması ile sağlanırsa fotolüminesans (FL) adı verilir. FL yoğunluğu, ölçüm sıcaklığına ve uyarma ışı̆̆ının enerjisine bağlıdır [4,5]. Absorpsiyon nedeniyle uyarılmış hale geçen atom veya moleküller için önerilen fotofiziksel prosesler Şekil 1'de Jablonski-Perrin diyagramı ile verilmiştir [3,4].

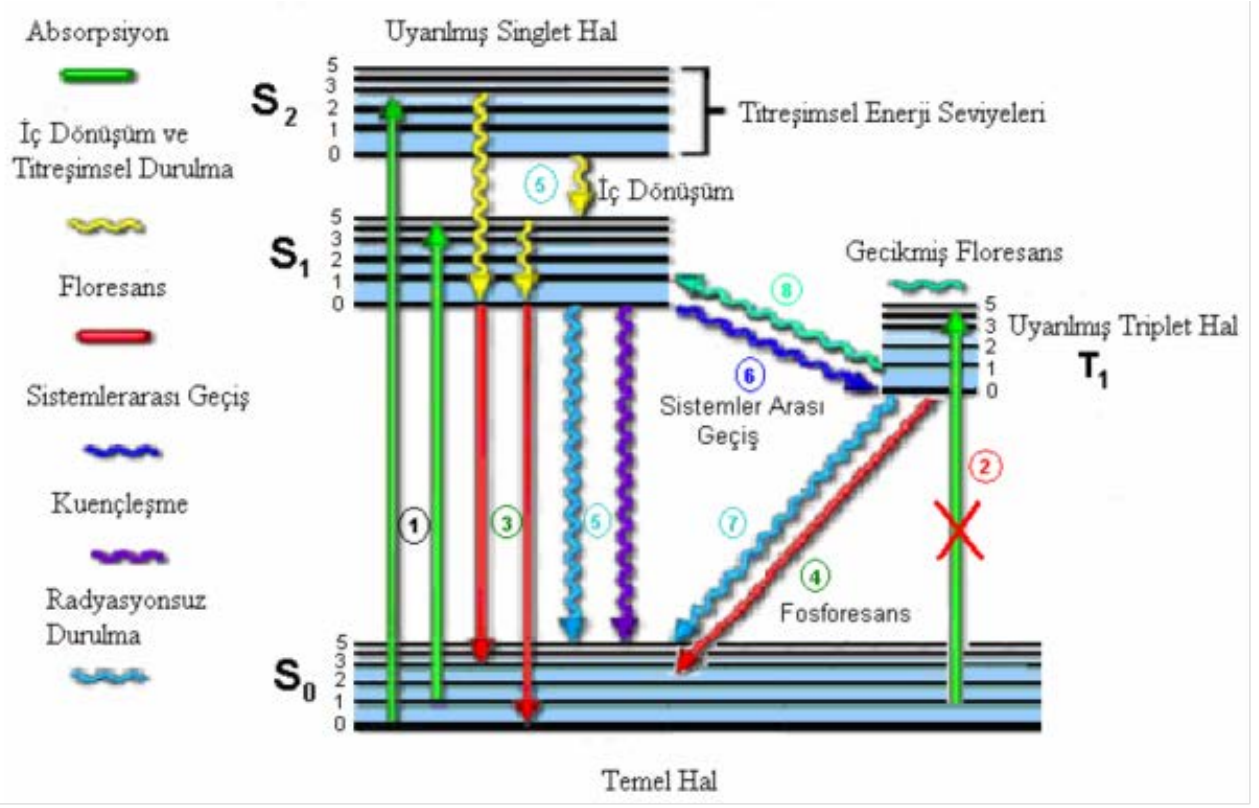

Şekil 1. Jablonski-Perrin Diyagramı

Fosfor malzemeler genellikle katı hal reaksiyonları ve sol-jel metoduyla sentezlenmektedir. Lüminesans malzemelerin üretim teknikleri ana başlıklar altında aşağıdaki gibi özetlenebilir.

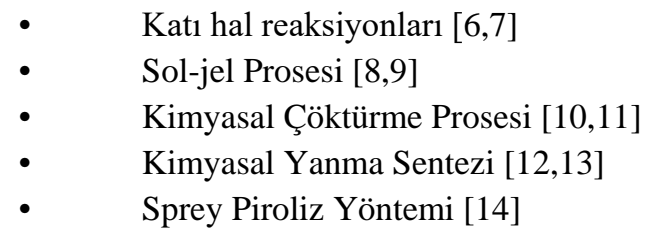

Endüstride birçok uygulama yüksek parlaklığa sahip ve uzun süreli ışıldama yapan malzemeleri kullanmakta veya ilgi duymaktadır. Uzun süre ışıyan fosforlar arasında yaklaşık 100 yıldır bilinen $\mathrm{ZnS}$ bazlı malzemeler çok geniş kullanım alanına sahiptir. Fakat bu malzemelerin düşük ışıma şiddeti ve süresi ile düşük kimyasal kararlılık özellikleri kullanım alanlarını sınırlandırmış ve yeni malzemelerin arayışına yol açmıştır. 1996 yılında Matzusawa ve 
arkadaşlarının çalışmalarından sonra, Eu ve Dy ile katkılandırılmış $\mathrm{SrAl}_{2} \mathrm{O}_{4}$ fosforuna olan ilgi artmıştır ve pek çok çalışmaya konu olmuştur [15]. Nadir toprak elementleriyle aktive edilmiş kalıcı fosforesans mekanizması tam olarak açıklanamasa da birkaç mekanizma önerilmiştir. Matsuzawa tarafindan önerilen mekanizma Jia ve diğerleri tarafından modifiye edilerek Şekil 2'de sunulmuştur [16,17].

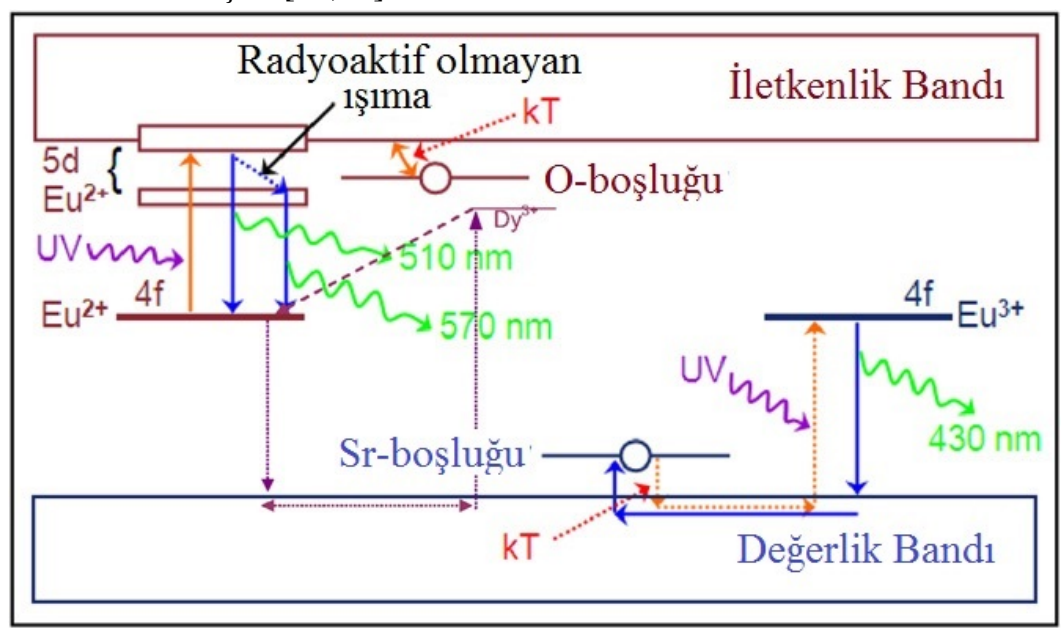

Şekil 2. Modifiye SrAl2O4: Eu2+, Dy3+ fosforesans mekanizması

Şekil 3'de görülmekte olan $\mathrm{SrO}-\mathrm{Al}_{2} \mathrm{O}_{3}$ ikili faz diyagramında; iyi bilinen $\mathrm{Sr}_{3} \mathrm{Al}_{2} \mathrm{O}_{6}, \mathrm{SrAl}_{2} \mathrm{O}_{4}, \mathrm{SrAl}_{4} \mathrm{O}_{7}$, $\mathrm{SrAl}_{12} \mathrm{O}_{19}$ anafazları bulunmaktadır ve istenen bileşim $\mathrm{SrO}$ ve $\mathrm{Al}_{2} \mathrm{O}_{3}$ 'ün molar oranlarının kontrol edilmesi ile elde edilmektedir [15,18]. Lüminesans maddelerin üretim teknikleri kısmında açıklanan bu yöntemlerde faz diyagramındaki birçok stronsiyum alüminat tozları elde edilmiştir. Bu yöntemlerin birbirine karşı avantaj ve dezavantajları bulunmaktadir.

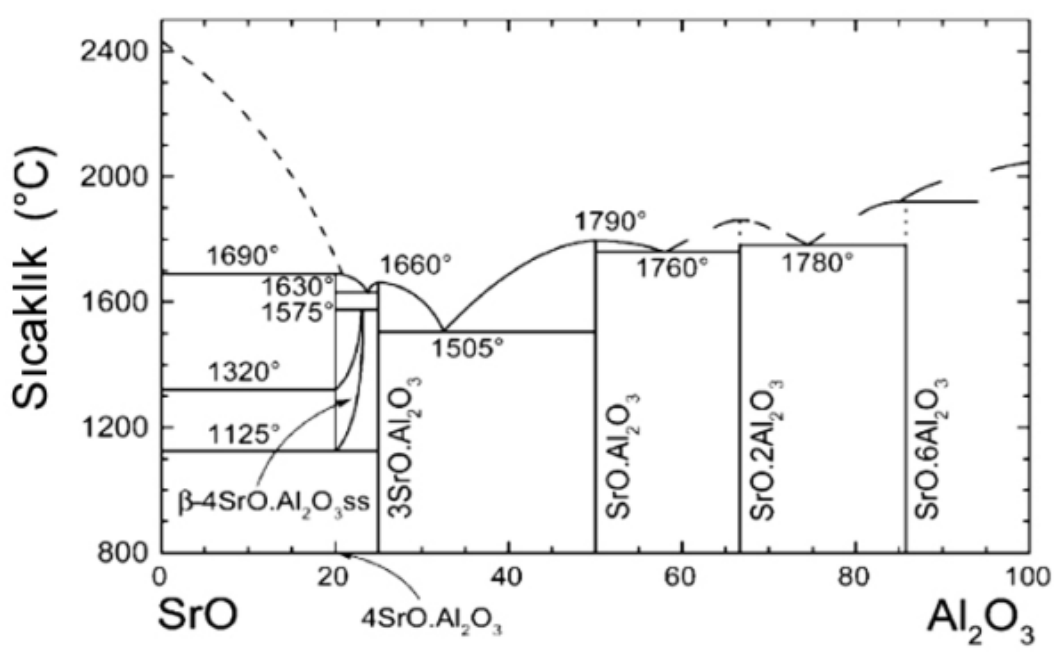

Şekil 3. SrO-Al2O3 ikili faz diyagramı

Kompozit malzeme, birbiri içinde çözünmeyen iki veya daha fazla malzemenin birleşmesine denir. Kompozit malzeme, kendisini oluşturan malzemelerin üstün özelliklerini birleştirerek her birinden farklı yeni bir tür malzemedir. Kompozit malzemeler, iki bileşenden meydana gelir; matris ve takviye malzemesi (Şekil 4). Genellikle takviye malzemesi fiber grubundan karbon, cam veya aramid olurken, taneli malzemelerden de oluşabilir ve dolgu veya filler olarak adlandırılır [19]. 


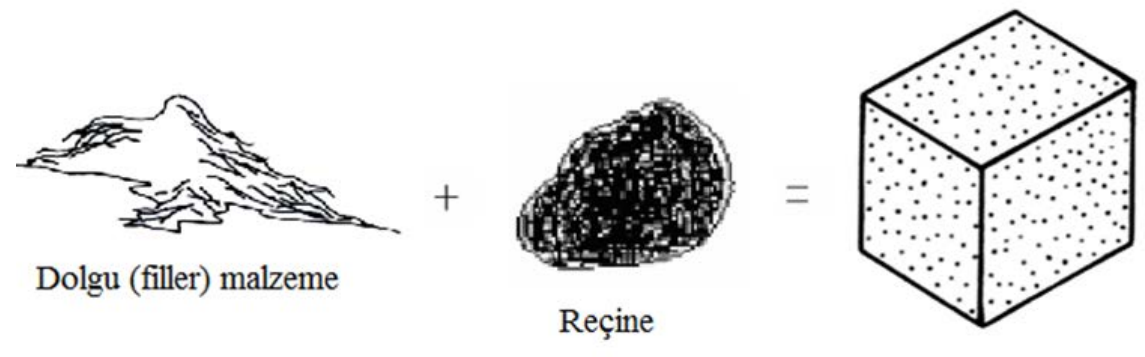

Taneli kompozit

Şekil 4 . Taneli kompozit malzemede

Kompozit malzemeler matris malzemesine göre üç grupta sınıflandırılırlar ;

- $\quad$ Polimer matrisli kompozitler (PMK)

- $\quad$ Metal matrisli kompozitler (MMK)

- $\quad$ Seramik matrisli kompozitler (SMK) [20-22].

Polimer matris kompozitler (PMK), düşük yoğunluk, dayanım ve aşınma gibi özelliklerinden dolayı birçok endüstriyel uygulamalarda tercih edilen malzeme olmuştur. Termoset polimerler mukavemeti ve yüksek sıcaklığa karşı dayanımları yönünden termoplastiklerden daha üstündür. Termoset polimerlerden olan Epoksi yapısı Şekil5'te verilmiş olup yaygın kullanılan polimerlerdendir [23]. Viskoz ve açık renkli bir sıvı halindedirler.

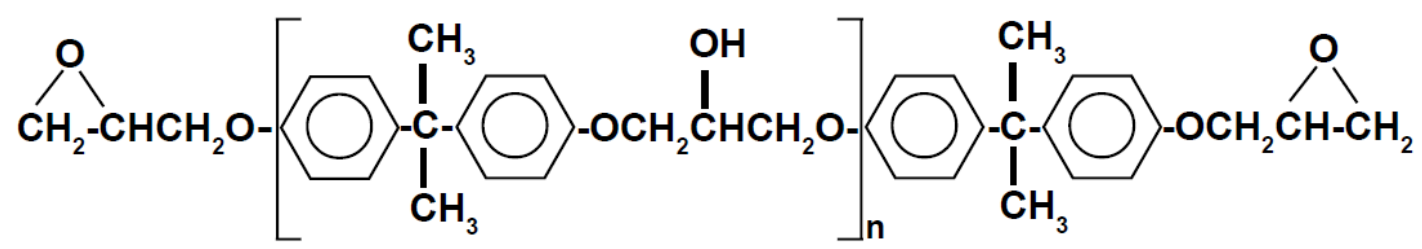

Şekil 5. Epoksi yapısı

Epoksilerin Avantajları:

- $\quad$ Kimyasal ve mekanik dayanımı yüksektir.

- $\quad$ Fiber destekli yapılarla yüksek bağ mukavemeti sağlar.

- Aşınmalara karşı dirençlidir.

- $\quad$ Kimyasal dirençleri yüksektir.

- $\quad$ Antistatik ve hijyeniktir.

Epoksilerin Dezavantajları

- $\quad$ Maliyetleri yüksektir.

- Doğru karışım çok önemlidir.

- $\quad$ Cilde aşırı zararlıdır [21-25].

$\mathrm{Bu}$ çalışmada Eu ve Dy katkılandırılmış Stronsiyum alüminat $\left(\mathrm{SrAl}_{2} \mathrm{O}_{4}: \mathrm{Eu}^{2+}, \mathrm{Dy}^{3+}\right)$ tozları sol-jel yöntemiyle hazırlanmış ve elde edilen tozlar literatürde ilk defa Epoksi reçinesi içerisine katılarak seramik bisküvi bir yüzey üzerine uygulanmış ve basit bir teknikle yüzeyde kendi kendine sşıldayan tabaka elde edilmiştir.

\section{Materyal ve Metot}

Deneysel çalışmalarda, Eu, Dy katkılı stronsiyum alüminatları $\left(\mathrm{SrAl}_{2} \mathrm{O}_{4}: \mathrm{Eu}^{2+}, \mathrm{Dy}^{3+}\right)$ üretebilmek için başlangıç kimyasalları olarak yüksek saflıkta (Merck \% 99,99) stronsiyum nitrat $\left(\operatorname{Sr}\left(\mathrm{NO}_{3}\right)_{2}\right)$, alüminyum nitrat $\left(\mathrm{Al}\left(\mathrm{NO}_{3}\right)_{3}\right)$, disprozyum nitrat $\left(\mathrm{Dy}\left(\mathrm{NO}_{3}\right)_{3}\right)$ ve evropiyum nitrat $\left(\mathrm{Eu}\left(\mathrm{NO}_{3}\right)_{3}\right.$ kullanılmıştır. Deney akım şeması Şekil 6'da verilmiştir. Deneysel çalışmalarda Tekno Yapı Kimyasalları A.Ş.' ne ait Teknobond 300 epoksi reçine kullanılmış olup teknik özellikleri Tablo 2'de verilmiştir. 
Nevşehir Bilim ve Teknoloji Dergisi (2018), 7(2) 156-165

\begin{tabular}{|c|c|}
\hline Özellik & Açıklama \\
\hline Yoğunluk & Karışım: $1,10 \pm 0,02(\mathrm{~kg} / \mathrm{L})$ \\
\hline Eğilme Mukavemeti ( 7 Gün ) & $>30 \mathrm{~N} / \mathrm{mm}^{2} \quad($ TS EN 196-1) \\
\hline Basınç Mukavemeti ( 7 Gün ) & $>75 \mathrm{~N} / \mathrm{mm}^{2} \quad($ TS EN 196-1) \\
\hline Betona Yapışma Mukavemeti & $>4 \mathrm{~N} / \mathrm{mm}^{2}$ (Betondan Kopma) ( TS EN 4624 ) \\
\hline Uygulama Süresi $\left(23^{\circ} \mathrm{C}, \% 50 \mathrm{Nem}\right.$ ) & 40 dak ( Hava şartlarına göre değişebilir ) \\
\hline Karışım Oranı & 2 Birim A: 1 Birim B (w\%) \\
\hline Tam Mukavemet & 7 Gün \\
\hline
\end{tabular}

Başlangıç kimyasalları herhangi bir saflaştırma işlemine tabi tutulmadan mutlak etil alkol ile çözeltileri hazırlanmış ve $\mathrm{SrAl}_{2} \mathrm{O}_{4}$ stokiyometrik bileşimlerine uygun şekilde karıştııılmıştır. Karışıma hazırlanan Dy ve Eu içeren çözeltiler (\% 0,01 mol) ilave edilip karıştırma işlemine devam edilmiştir. Çözelti $60^{\circ} \mathrm{C}$ 'de 6 saat karıştırılmış ve beyaz jel formu elde edilmiştir. $180{ }^{\circ} \mathrm{C}$ 'de 24 saat kurutulduktan sonra $1200{ }^{\circ} \mathrm{C}$ 'de 4 saat kalsine edilmiştir. Elde edilen tozların öğütülmüş hali normal ışıkta, karanlıkta ve UV ışıkta Şekil 7'de görülmektedir.

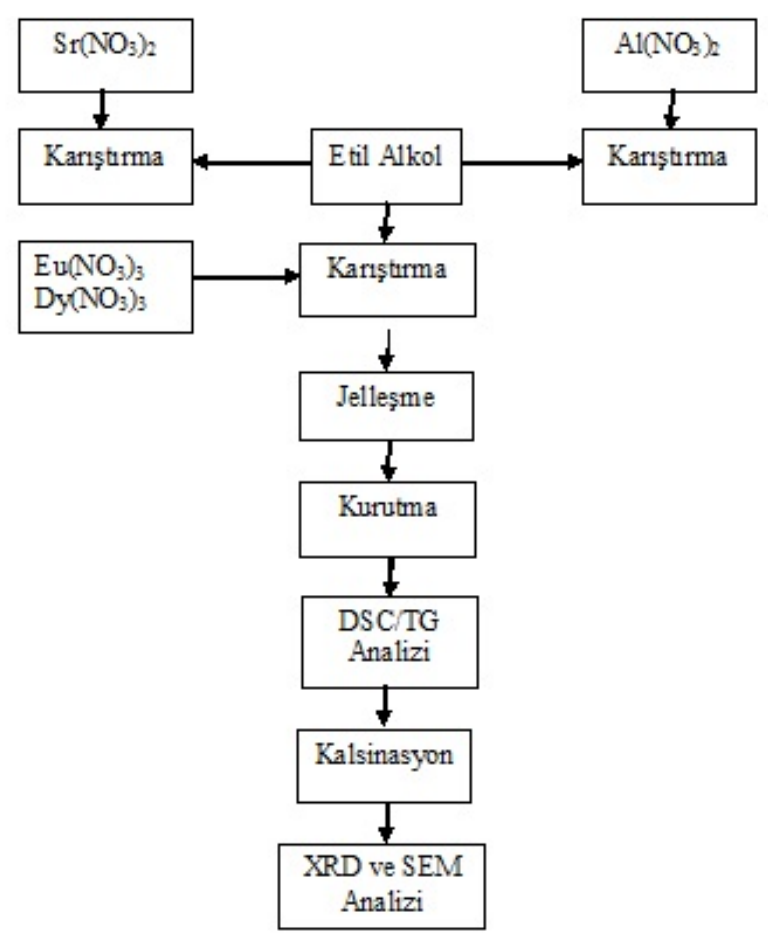

Şekil 6. Deney akım şeması

Elde edilen tozlara uygulanacak ısıl işlem sıcaklığını belirlemek ve termal davranışlarını saptamak amacıyla stronsiyum alüminyum oksit bileşimine $10^{\circ} \mathrm{C} / \mathrm{dk}$ ssıtma hızı ile azot atmosferinde $1300{ }^{\circ} \mathrm{C}$ sıcaklığa kadar, TG/DSC analiz tekniği NETZSCH STA 449 F3 JUPITER cihazı ile test edilmiştir. Mineralojik yapısını görmek amacıyla SHIMADZU XRD-6000 markalı X-Işınları Kırınımı (XRD) cihazı ile ( $\lambda=1,5406 \AA$, $\theta: 20^{\circ}$ ile $70^{\circ}$ arası) karakterizasyonu yapılmıştır. Morfolojik yapısı ise LEO 1430 VP SEM (taramalı elektron mikroskop) ile incelenmiştir. 


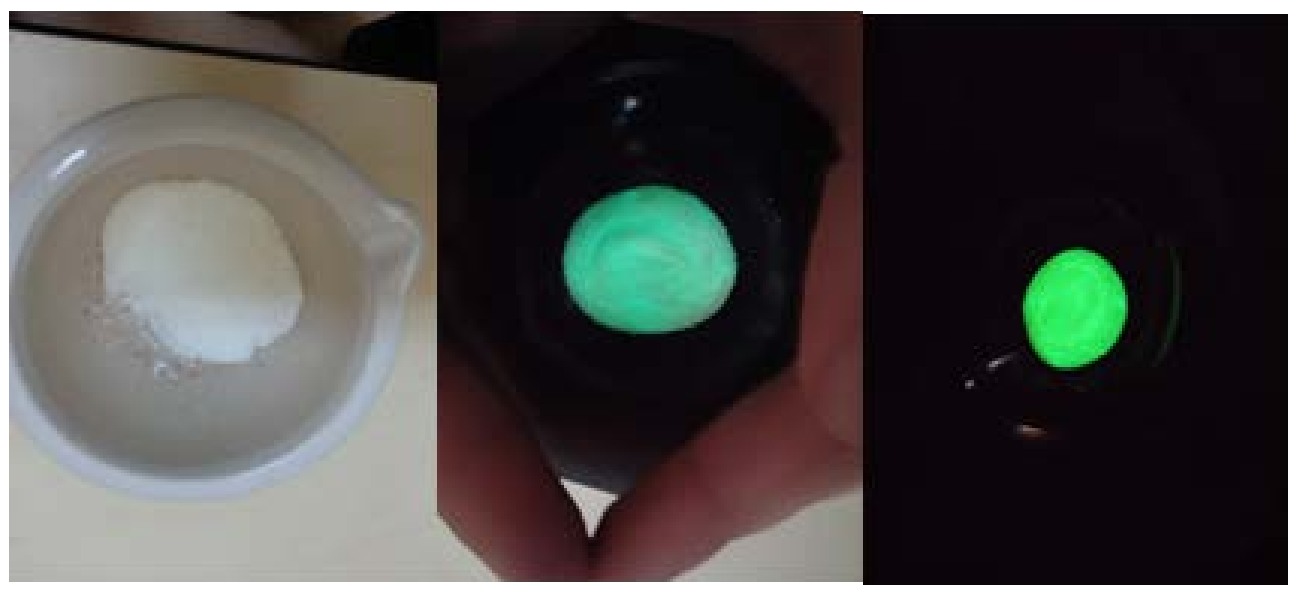

Şekil 7. Elde edilen tozlar

Epoksi reçine teknik özelliklerinde de belirtildiği gibi ağırlıkça \% 2 birim A ve 1 birim B olacak şekilde plastik derin bir kaba dökülerek 10 dakika karıştırılmıştır. Bu Epoksi karışımı ikiye bölünerek yarısına ağırlıkça \% $10 \mathrm{SrAl}_{2} \mathrm{O}_{4}$ : Eu, Dy tozları katılarak 1 dak. karıştırılmış, diğer yarısı katkısız olarak ayrılmıştır. Ön pişirimi yapılmış desenli seramik bisküvi üzerinde istenen bölgeler bu $\mathrm{SrAl}_{2} \mathrm{O}_{4}: \mathrm{Eu}^{2+}, \mathrm{Dy}^{3+}$ toz katkılı karışım kalem fırça ile uygulanmış, diğer bölgeler ise katkısız ayrılmış olan Epoksi ile kaplanmıştır. Bu sayede hem kolay uygulamaya sahip, ileri teknoloji gerektirmeyen, karmaşık şekillerdeki malzemelerin uzun süreli 1şıldayan maddelerle kaplanabilmesi sağlanmıştır. Kaplanmış yüzeyle kendi haline oda sıcaklığında kurumaya bırakılmıştır.

\section{Bulgular}

Şekil 8'de tozlara uygulanan TG/DSC analiz sonucu görülmektedir.

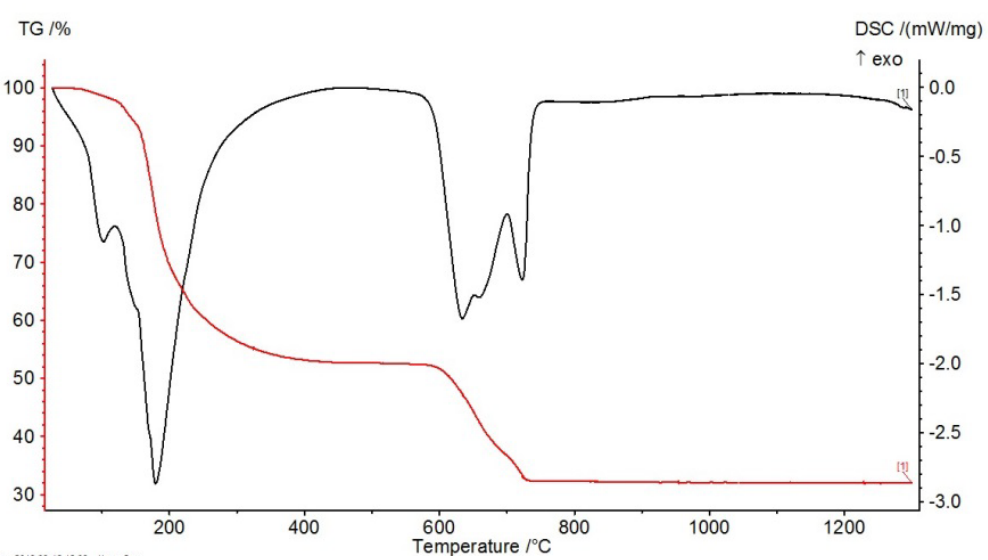

Şekil 8. Evropiyum-Disprozyum Katkı1ı Stronsiyum Alüminyum Oksit Tozunun TG/DSC Analizi

TG analizinde $500^{\circ} \mathrm{C}$ 'ye kadar $\% 45,27 ; 600^{\circ} \mathrm{C}^{\prime}$ den $1300^{\circ} \mathrm{C}$ 'ye kadar ise \% 20,16 kadar ağırlık kaybı gözlenmiştir. DSC analizinde $82^{\circ} \mathrm{C}^{\prime} \mathrm{de}-0,9645 \mathrm{~mW} / \mathrm{mg}$ ve $168,7{ }^{\circ} \mathrm{C}$ 'de $-2,676 \mathrm{~mW} / \mathrm{mg} 633,3^{\circ} \mathrm{C}$ 'de $-1,892 \mathrm{~mW} / \mathrm{mg}$ 656,0 ${ }^{\circ} \mathrm{C}$ 'de $-1,686 \mathrm{~mW} / \mathrm{mg} 697,4{ }^{\circ} \mathrm{C}$ 'de $-1,469 \mathrm{~mW} / \mathrm{mg}$ endotermik ve 947,6 ${ }^{\circ} \mathrm{C}$ 'de $0,04048 \mathrm{~mW} / \mathrm{mg}$ ekzotermik pik bulunmuştur.

25-168,7 ${ }^{\circ} \mathrm{C}$ arasındaki sıcaklıklardaki meydana gelen büyük ağırlık kaybı ve endotermik reaksiyon yine başlangıç maddelerinin içerdiği nem, organik uçucu bileşen ve çözücülerin uzaklaşmasından meydana gelmiştir. 633,3$656,0{ }^{\circ} \mathrm{C}$ sıcaklık aralığındaki diğer endotermik pikler $\mathrm{Al}$ ve $\mathrm{Sr}$ hidroksitlerin termal dekompozisyonunu göstermektedir, meydana gelen ağırlık kayıpları da bunu desteklemektedir. 


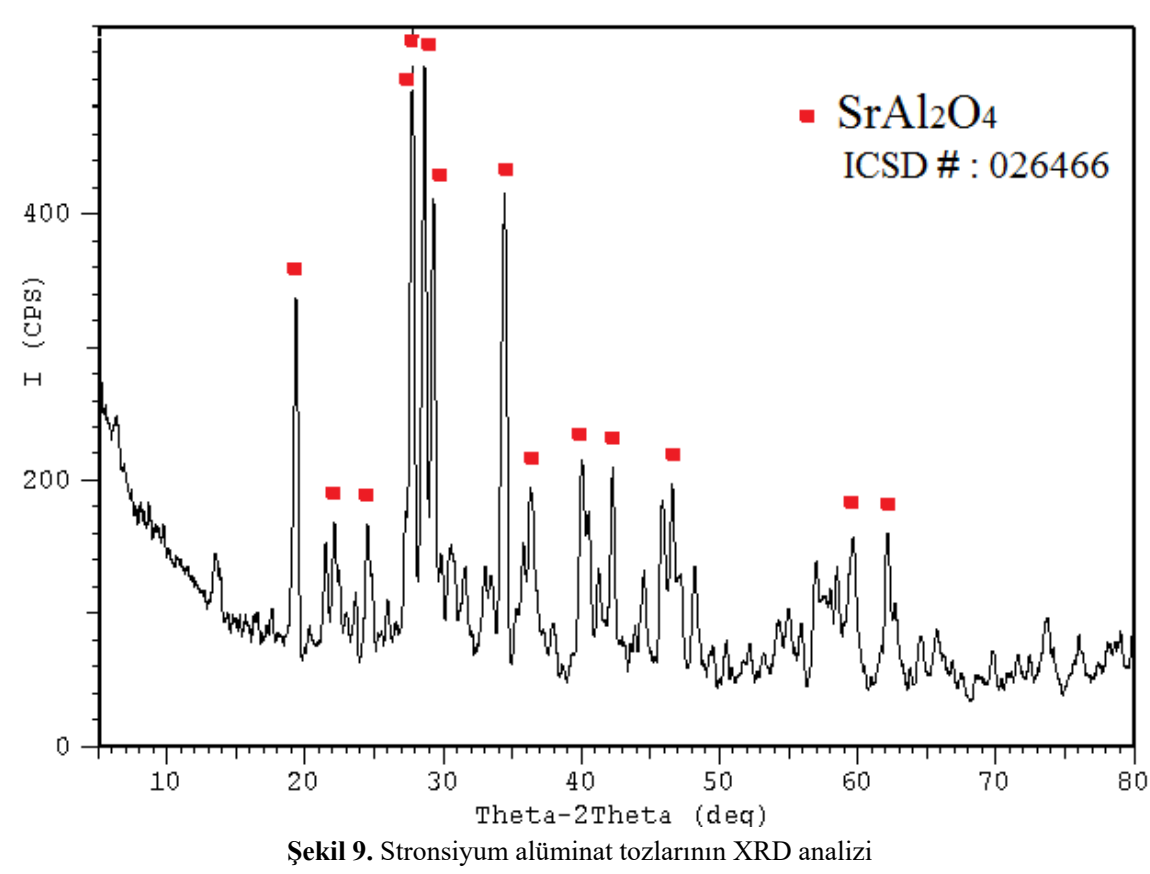

Şekil 9'da görüldüğü gibi üretilen 0,01 mol Eu-Dy katkılı tozların XRD analizinde literatürle benzer sonuçlar elde edilmiş ve tozun monoklinik ( $\mathrm{a}=8.447 \mathrm{~b}=8.816 \mathrm{c}=5.163) \mathrm{SrAl}_{2} \mathrm{O}_{4}$ 'e ait olduğu (ICSD \# : 026466) belirlenmiştir [4,15]. Katkı miktarı molce \% 1 olduğundan XRD paternlerinde Eu ve Dy'ye ait bileşiklerin pikleri görülmemiştir. Şekil 10'daki toz haline getirilmiş numuneye ait morfolojik analizi görülmektedir.

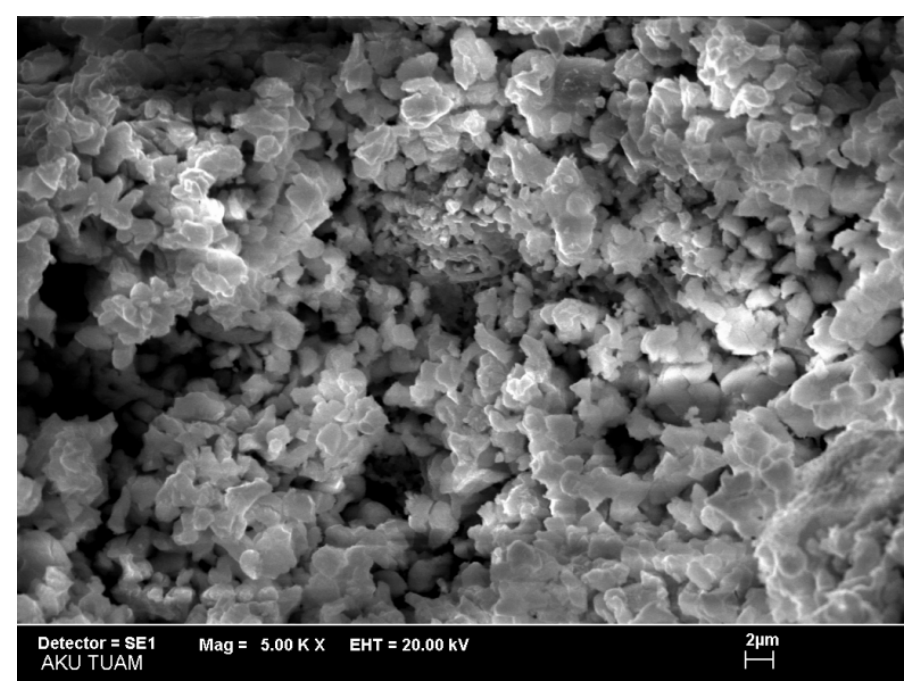

Şekil 10. Üretilen tozların SEM fotoğrafı

Şekil 10'da görüldüğü gibi genel yapının $1 \mu \mathrm{m}$. boyutlarında, belirgin bir şekle sahip olmayıp küresele yakın formlarda tanelerden oluştuğu ve homojen bir dağılım gösterdiği tespit edilmiştir.

Elde edilen tozlar epoksi reçine ile karıştırılmış ve bisküvi pişirilmiş seramik yüzeyler üzerine uygulanmıştır (Şekil 11). Desenli yüzey üzerine uygulanmış $\mathrm{SrAl}_{2} \mathrm{O}_{4}: \mathrm{Eu}^{2+}, \mathrm{Dy}^{3+}$ tozu katkılı Epoksi bölgesi diğer katkısız epoksili bölgeden tamamen farklı bir ışıldamaya sahiptir. 10 dakika güneş ışı̆̆ına bırakılmış seramik yüzey karanlık bir ortamda şekildeki gibi ışıldama göstermektedir. 


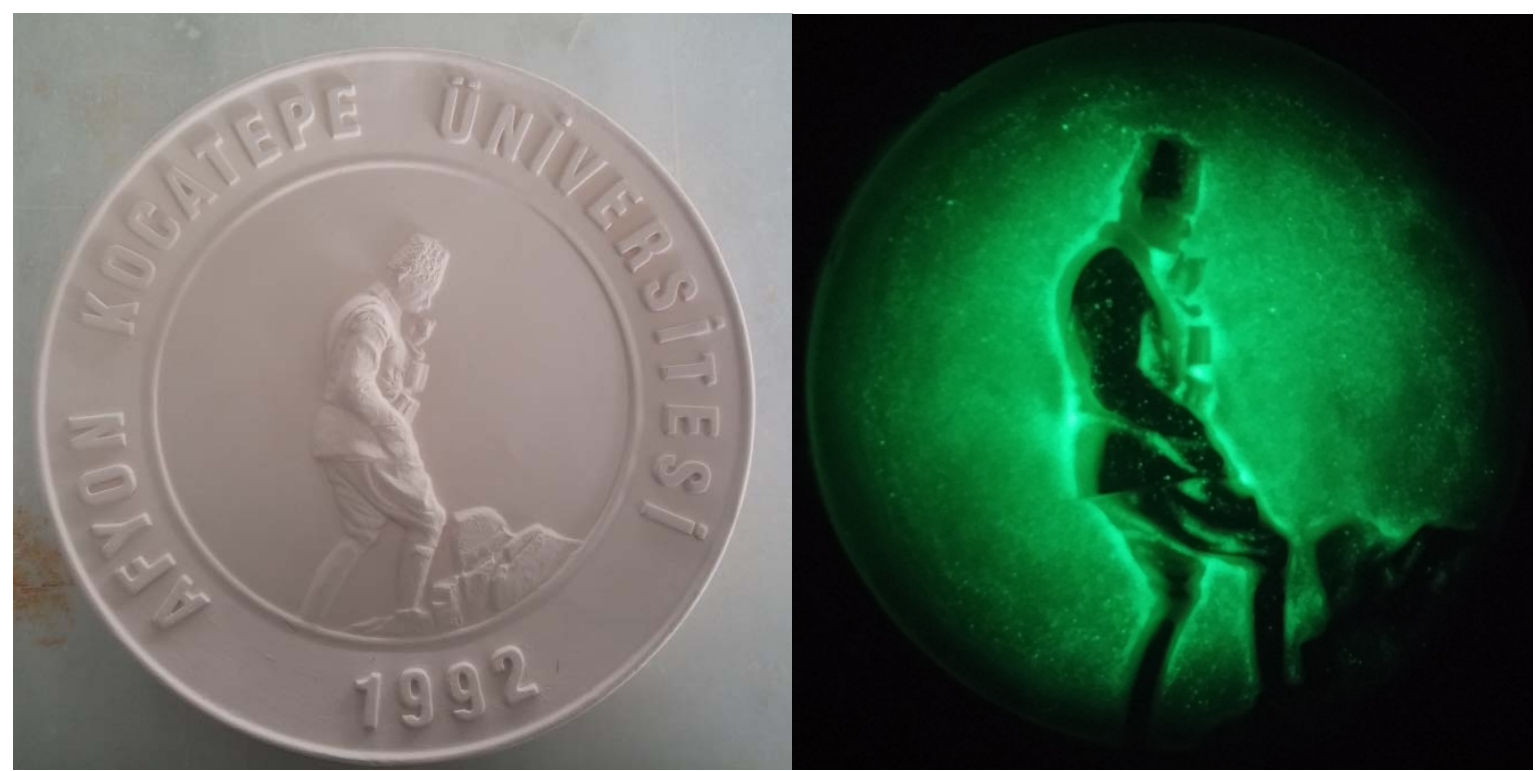

Şekil 11. Yüzeylere uygulanan SrAl2O4: $\mathrm{Eu}^{2+}, \mathrm{Dy}^{3+}$ fosforu ve epoksi kompoziti.

\section{Tartışma ve Sonuç}

Sonuç olarak birçok endüstri alanında büyük öneme sahip lüminesans malzemelerden, uzun süre 1şıldama yapan $\mathrm{SrAl}_{2} \mathrm{O}_{4}: \mathrm{Eu}^{2+}$, $\mathrm{Dy}^{3+}$ fosforu ileri teknoloji yöntemlerinden sol-jel prosesiyle düşük sıcaklıkta üretilmiştir. Monoklinik yapıda üretilmesi amaçlanan bu tozların termoset bir reçine olan Epoksi içerisine eklenerek seramik bisküvi üzerine uygulanması neticesinde ışıldama yapan kompozit yüzey ilk defa üretilmiştir. Sadece termoset değil termoplastik polimerlere de kolaylıkla karıştırılabilen $\mathrm{SrAl}_{2} \mathrm{O}_{4}: \mathrm{Eu}^{2+}, \mathrm{Dy}^{3+}$ içeren kompozitler her türlü düz, pürüzlü, desenli veya karmaşık yüzeylere uygulanabilir. Bunun için ne bir yüksek sıcaklık ne de basınç gibi bir etken kullanılmaksızın kısa bir süre içerisinde yüzeylere uygulanabilmesi, daha önceden uygulanmış yüzeylerin onarılması en büyük avantajlarındandır.

\section{Tessekkür}

$\mathrm{Bu}$ çalışma Afyon Kocatepe Üniversitesi Bilimsel Araştırma Projeleri Komisyonu (No: 13.MUH.02 ve 17.KARIYER.87) tarafindan desteklenmiştir.

\section{Kaynaklar}

[1] Kaya S. Y. and Karasu B., "Mavimsi-yeşil ve sarımsı-yeşil fosforesans pigmentlerin üretimi, duvar karosu vetroza uygulamalarında kullanımı”, Seramik Türkiye, pp 2-8, Ocak-Mart 2010

[2] Kasap, S., Capper, P.,”Electronic and photonic materials”, Springer, New York, 2006

[3] Albani J.R., "Principles and Applications of Fluorescence Spectroscopy”, Blackwell publishing, France, 2007

[4] Karakaş C., Modifiye Edilmiş Sol-jel Prosesiyle Stronsiyum Alüminat Esaslı Fosforesans Malzeme Sentezi, Yüksek Lisans Tezi, İTÜ, İstanbul, Haziran 2010

[5] Uluç A.V., Synthesis and characterization of phosphorescent strontium aluminate, Msc Thesis, Sabanc1 Üniversitesi, İstanbul, 2008

[6] Sahu I.P., Bisen D.P., Brahme N., Tamrakar R.K., "Luminescence behavior of europium activated strontium aluminate phosphors by solid state reaction method”, Journal of Materials Science: Materials in Electronics, Volume 27, Issue 4, pp 3443-3455, April 2016

[7] Minquan W., Wang D., Guanglie L., "Research on Fluorescence Spectra and Structure of Single-phase 4SrO-7Al2O3: Eu2+ Phosphor Prepared by solid-State Reaction Method”, Material Science and Engineering, B57, 18-23, 1998.

[8] Yuesheng C., Ping Z. and Zhentai Z., "Eu2+ and Dy3+ Co-doped Sr3Al2O6 Red Long-Afterglow Phosphors With New Flower-Like” Morphology, Physica B., 403, 2008. 
[9] Misevicius M., Scit O., Grigoraviciute-Puroniene I., Degutis G., Bogdanoviciene I., Kareiva A., "Sol-gel synthesis and investigation of un-doped and Ce-doped strontium aluminates”, Ceramics International Volume 38, Issue 7, pp 5915-5924, September 2012

[10]Chang C., Yuan Z., Mao D., "Eu2+ activated long persistent strontium aluminate nano scaled phosphor prepared by precipitation method”, Journal of Alloys and Compounds 415, pp 220-224., 2006

[11] Rojas-Hernandez R. E., Rodriguez M. A., Rubio-Marcos F., Serrano A.and Fernandez J. F., "Designing nanostructured strontium aluminate particles with high luminescence properties”, Journal of Materials Chemsitry C, İssue 6,2015

[12] Montes-Frausto J. B., Juarez-Moreno K., Can-Uc B., and Hirata-Flores G. A., "Synthesis and cytotoxic effects of SrAl2O4 persistent luminescence nanoparticles co-doped with Eu2+=Dy3+ ions”, OSA Optical Society of America OPTICAL MATERIALS EXPRESS, Vol. 6, No. 5, 1 May 2016

[13] Song H., Chen D., Tang W., Peng Y., “Synthesis of SrAl2O4: Eu2+, Dy3+, Gd3+ Phosphor by Combustion Method and Its Phosphorescence Properties”, Elsevier, 29, 41-44, 2008.

[14] Kim M.N., Jung K.Y., "Synthesis and Luminescence Enhancement of Strontium Aluminate Green Phosphor via Spray Pyrolysis”, Korean Chemical Engineering Research 49 (5) October 2011

[15] Arıkan M.O., Katı Hal Reaksiyonuyla Stronsiyum Aluminat Esaslı Fosforesans Malzeme Sentezi, Yüksek Lisans Tezi, İTÜ, İstanbul, 2010

[16] Luitel H.N. , Preparation and Properties of Long Persistent Sr4Al14O25 Phosphors Activated by Rare Earth Metal Ions, PhD Thesis, Saga University, March 2010

[17] Swart H.C., Terblans J.J., Ntwaeaborwa O.M., Kroon R.E., Mothudi B.M., PL and CL degradation and characteristics of SrAl2O4: Eu2+, Dy3+ phosphors, Physica B: Condensed Matter 407 (10), 1664-1667 2012

[18]Zurba N. K., Persistent luminescence of SrAl2O4:Ce(III), Dy, Eu nanotubes, nanowires and core-shells for people with disabilities: nano-emergency, PhD Thesis, Universidade de Aveiro, 2010

[19] EVCIN A., GÜNEŞ İ., UYGUNOĞLU T., ERSOY B., “Kireçtaşı Katkı1ı Epoksi Zemin Kaplamaların Yüzey Özellikleri”, International Conference on Natural Science and Engineering (ICNASE’16), pp 3443-3450, Kilis, March 19-20, 2016

[20] Gökkaya İ. Tek Bindirmeli Örgülü Kompozit Malzemelerin Darbe Davranışının İncelenmesi, Pamukkale Üniversitesi, Yüksek Lisans Tezi, Denizli, (2015), pp 8-11

[21] Evcin A. Polimer Malzemeler Ders Notları, Afyon Kocatepe Üniversitesi, (2016)

[22] Demirel A., Karbon Elyaf Takviyeli Kompozit Malzemelerin Karakterizasyonu, Gazi Üniversitesi, Yüksek Lisans Tezi, Ankara (2007)

[23] Augustson C. N.M., Epoxy Handbook, Nils Malmgren AB, Sweeden, 2004.

[24] Saçak M. Polimer Teknolojisi, Gazi Kitabevi, Ankara (2005), pp. 219-228

[25] Lee S.B., Lee H.J., Hong I. K, (2012) Diluent filler particle size effect for thermal stability of epoxy type resin, Journal of Industrial and Engineering Chemistry, 18, 635-641 
Nevşehir Bilim ve Teknoloji Dergisi (2018), 7(2) 156-165

\section{Extended Abstract}

\section{Introduction}

Luminescent materials have attracted much attention. One of the luminescent materials is the Strontium Aluminate. From the phase diagram of SrO and $\mathrm{Al}_{2} \mathrm{O}_{3}, 5$ different binary phases $\left(\mathrm{Sr}_{4} \mathrm{Al}_{2} \mathrm{O}_{7}, \mathrm{Sr}_{3} \mathrm{Al}_{2} \mathrm{O}_{6}, \mathrm{SrAl}_{2} \mathrm{O}_{4}, \mathrm{SrAl}_{4} \mathrm{O}_{7}\right.$ ve $\left.\mathrm{SrAl}_{2} \mathrm{O}_{19}\right)$ can be obtained. Among them, $\mathrm{SrAl}_{2} \mathrm{O}_{4}$ has excellent chemical stability and long-lasting luminescence. Different additives have been used to have more luminescence. Among these additives, Eu and Dy are the most effective and rare earth elements. $\mathrm{SrAl}_{2} \mathrm{O}_{4}$ powders are synthesized by solid state reaction, sol-gel method, microwave heating, chemical combustion method, laser melting method, flame spray pyrolysis, chemical precipitation method and Pechini Process. Sol-gel method is a wet chemical process. In the sol-gel process, the precursors are metal alkoxide (M[OR]n , M:metal R:alkyl and n: valence of metal) and metal salts (MX, M.metal and X:anion). The main advantages of this method are high purity, small sized powder, low temperature, control of composition, large surface area, and applicable to much industry. Many different form can be achieved such as powder, monoliths, fibers, coatings and thin films, porous materials and aerogels by sol-gel method.

\section{Method}

In this study, Eu-Dy doped strontium aluminate powders were prepared by sol-gel method. To fabricate $\mathrm{SrAl}_{2} \mathrm{O}_{4}$, proper chemical precursors (Strontium nitrate, Aluminum nitrate) were chosen. The precursor materials used were analytical grade and supplied from Merck A.G. Aluminum nitrate [Al(NO3)3] and strontium nitrate [Sr(NO3)2] were both dissolved in ethyl alcohol and they were vigorously mixed for $1 \mathrm{~h}$ at ambient temperature. All chemicals were used as received. Then their solutions were prepared. The nitrates of Eu and Dy $\left[\mathrm{Dy}\left(\mathrm{NO}_{3}\right)_{3}\right.$ and $\left.\mathrm{Eu}\left(\mathrm{NO}_{3}\right)_{3}\right]$ were both dissolved in $1 \mathrm{~mL}$ deionized water, and then both solutions were mixed. $0.01 \mathrm{~mol}$ of Dysprosium nitrate and Europium nitrate was added to the solution formed by mixing their solutions. Gelling powders at room temperature were dried in the oven at $180^{\circ} \mathrm{C}$ for 24 hours. Dried powders were sintered at $1200^{\circ} \mathrm{C}$ for 4 hours. In order to determine the characteristics of polymer matrix composites, epoxy consisting of two components in A and B (2: 1) was used in the production of the samples. The density of the epoxy components was $1.15 \mathrm{~kg} / \mathrm{dm}^{3}$ of component A and $1.05 \mathrm{~kg} / \mathrm{dm}{ }^{3}$ of component B, respectively. The color of epoxy resin was transparent. The minimum application temperature was $10^{\circ} \mathrm{C}$. In addition, the usage time of $20{ }^{\circ} \mathrm{C}$ was 45 minutes. In the polymer mixture, the component $\mathrm{A}$ of the polymer resin with $\mathrm{SrAl}_{2} \mathrm{O}_{4}: \mathrm{Eu}^{2+}, \mathrm{Dy}^{3+}$ powders was stirred for 1 min. The polymer was mixed with the component $\mathrm{B}$ of the polymer resin until homogenous. $\mathrm{SrAl}_{2} \mathrm{O}_{4}: \mathrm{Eu}^{2+}, \mathrm{Dy}^{3+} /$ Epoxy composite was applied on ceramic surfaces. After $\mathrm{SrAl}_{2} \mathrm{O}_{4}: \mathrm{Eu}^{2+}, \mathrm{Dy}^{3+} /$ Epoxy thin film was dried, luminescence properties of thin film was examined in the dark.

\section{Results and Discussion}

Thermal, morphological and crystallographic properties of SrAl2O4:Eu2+,Dy3+ powders were characterized by using thermogravimetric/differential scanning calorimetry (DSC/TGA), X-ray diffraction (XRD) and scanning electron microscopy (SEM) techniques, respectively. It was observed that the powders were of SrAl2O4 structure and no undesirable phase was present. In the XRD pattern of the powder, the characteristic peaks of SrAl2O4 phase are very sharp. It was observed that the applied composite showed long-lasting luminescence. Powders having a particle size distribution of less than $1 \mu \mathrm{m}$ were obtained by sol-gel method. It is also seen from the SEM photograph where the particle size distribution is within a narrow range. To understand the thermal properties of $\mathrm{SrAl} 2 \mathrm{O} 4$ on the formation of powders during rasing temperature, DSC/TGA test were examined. There are two distinct stages of weight loss when temperature increases in DSC/TGA graphic. The TG curve reveals no weight loss after $750^{\circ} \mathrm{C}$ indicating the thermal decomposition of $\mathrm{SrAl} 2 \mathrm{O} 4$ have finished and the crystallization of $\mathrm{SrAl} 2 \mathrm{O} 4$ completed. The green $\mathrm{SrAl}_{2} \mathrm{O}_{4}$ : $\mathrm{Eu}^{2+}$, $\mathrm{Dy}^{3+}$ phosphors was successfully synthesized by sol-gel process. $\mathrm{SrAl}_{2} \mathrm{O}_{4}: \mathrm{Eu}^{2+}, \mathrm{Dy}^{3+} /$ Epoxy composite can be easily applied to different surfaces such as glass, polymer, metal and wood without requiring high technology. 